\title{
Comportamiento de la columna total de ozono obtenidos por el sensor Eptoms de la NASA para la ciudad de Puno periodo 2005-2017
}

\author{
Behavior of the Total Ozone Column obtained by NASA's Eptoms for the city of Puno, \\ 2005-2017 \\ Ciro William Taipe Huaman ${ }^{1}$, Matias Huillca \\ Arbieto $^{2}$, Eva Genoveva Mendoza Mamani ${ }^{1}$, \\ Julio Rumualdo Gallegos Ramos ${ }^{1}$ \\ ${ }^{1}$ Docente de la Universidad Nacional de Juliaca, Puno, Perú. \\ Email: c.taipe@unaj.edu.pe. \\ ${ }^{2}$ Docente de la Universidad Nacional del Altiplano Puno, Perú. \\ Email: mhuillca@unap.edu.pe \\ ${ }^{1}$ Docente de la Universidad Nacional de Juliaca, Puno, Perú \\ Email: genova2005@hotmail.com \\ ${ }^{2}$ Docente de la Universidad Nacional del Altiplano Puno, Perú. \\ Email: juliogallegos01@gmail.com
}

\section{OPEN ACCESS}

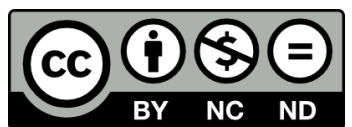

Copyright:(C) 2019 Ingenierías USBmed. La revista Ingenierías USBmed proporciona acceso abierto a todos sus contenidos bajo los términos de la licencia creative commons Atribución- no comercial- SinDerivar 4.0 Internacional (CC BY-NC-ND 4.0)

Tipo de Artículo: Investigación científica y tecnológica.

Recibido:21-04-2019.

Revisado: 11-06-2019.

Aprobado: 24-06-2019.

Doi: $10.21500 / 20275846.4057$

Referenciar así: C.W. Taipe, M. Huillca, E.G. Mendoza \& J.R. Gallegos. "Comportamiento de la columna total de ozono obtenidos por el sensor Eptoms de la NASA para la ciudad de Puno periodo 2005-2017". Ingenierías USBMed, 10(2), pp.25-30, 2019.

Declaración de disponibilidad de datos: Todos los datos relevantes están dentro del artículo, así como los archivos de soporte de información.

Conflicto de intereses: los autores han declarado que no existen conflicto de intereses.

Editores: Yohana López Rivera, Universidad de San Buenaventura, Medellín, Colombia. Alfonso Insuasti Rodríguez, Universidad de San Buenaventura, Medellín, Colombia. Erika Solange Imbett Vargas, Instituto Tecnológico Metropolitano. Eliana Zapata Ruiz, Instituto Tecnológico Metropolitano. José Fernando Valencia Grajales, Universidad Autónoma Latinoamericana.
Resumen. En este trabajo se han analizado datos de la columna total de ozono derivados de las mediciones realizadas por el Espectrómetro de Mapeo de Ozono Total de la Sonda de la Tierra (Eptoms) de la Administración Nacional de Aeronáutica y del Espacio (NASA), valorando su idoneidad para la predicción del índice ultravioleta (UV) para la ciudad de Puno $\left(15.83^{\circ}\right.$ latitud Sur y $70.03^{\circ}$ longitud Oeste). Al realizarse un análisis por año mediante ajustes de funciones senoidal, se observa que el desfasaje varía de un año a otro. Asimismo, durante el periodo estudiado (2005-2017) se encontraron tres fechas en las cuales se presentaron agujeros de ozono: el 5 de febrero de 2010 y el 29 de abril del 2016 se presentaron valores de 218 unidades Dobson (UD); y el 17 de mayo de 2005 se presentó el valor de 219 UD. El comportamiento de columna de ozono por año ostenta una forma cíclica senoidal, como lo establece el ciclo estacional. Palabras Clave. Ozono, índice UV, sensor eptoms, puno, NASA.

Abstract. Data of the Total Ozone Column have been analyzed in this paper, which were derived from the measurements performed by the Earth Probe Total Ozone Mapping Spectrometer (EPTOMS) of the National Aeronautics and Space Administration (NASA), by assessing its suitability for the prediction of the Ultraviolet (UV) Index for the city of Puno $\left(15.83^{\circ}\right.$ south latitude and $70.03^{\circ}$ west longitude). After a one-year analysis was performed by means of adjustments of sinusoidal functions, it is observed that the gap changes, from one year to the next. Additionally, during the period studied (2005-2017), three dates were found in which ozone holes were presented: On February 5, 2010 and April 29, 2016, values of 218 Dobson Units (DU) were presented, and on May 17,2005 , a value of 219 DU was presented. The behavior of the ozone column, per year, displays a sinusoidal cyclical shape, as it is established in the seasonal cycle.

Keywords. Ozone, UV index, eptoms sensor, puno, NASA. 


\section{Introducción}

La exposición excesiva a la radiación solar, y de modo específico, a la ultravioleta, ha sido causa de diferentes enfermedades, en especial de cáncer en la piel [1,2]. En este sentido, el índice ultravioleta (UV por sus iniciales en inglés) es un parámetro creado para efectos de divulgación y prevención que da cuenta de la irradiancia eritemática de un modo sencillo [3]. Así, se ha constatado la necesidad de informar y concienciar a la opinión pública de los riesgos que conlleva una excesiva exposición solar, y de la necesidad de aplicar medidas de fotoprotección [4].

La predicción de la radiación solar ultravioleta en la ciudad de Puno para días claros se realiza mediante el método paramétrico de la radiación solar ultravioleta [5, 6]. Este último requiere el ingreso de datos específicos para utilizarse: fecha, hora, longitud, latitud del lugar, altura del terreno sobre el nivel del mar y valor de la columna total de ozono (TOC por sus iniciales en inglés) en unidades Dobson (UD). Para cielos claros, el factor atenuante que más influye en la radiación solar ultravioleta es la TOC [4, 7], por lo que su estimación adecuada determinará en buena medida una óptima predicción del índice UV.

La TOC puede calcularse a través de un ozonosondeo, o mediante un instrumento llamado espectrofotómetro Brewer, que mide el ozono total en columna en UD. En la ciudad de Puno no existe un registro de TOC, por lo que se hace necesario recurrir a los datos del instrumento Total Ozone Mapping Spectrometer (TOMS) que se encuentran a bordo de los satélites NimBus-7 [8, 9]; los datos se encuentran disponibles en la Web. En este trabajo realizamos una muestra de la variación de la columna total de ozono para la ciudad de Puno en el periodo 2005-2017.

El TOC varía de forma natural en diferentes escalas de tiempo — anual, estacional, etc. - y de modo cíclico. Estas variaciones obedecen a diversos fenómenos naturales, entre los que se encuentran los ciclos solar y el mencionado estacional [10]; mientras que en otros responden a fenómenos naturales menos predecibles como erupciones volcánicas, el fenómeno del Niño, etc. [8]. Sumado a ello, y sin sombra de duda, la actividad humana afecta el contenido total de ozono en la atmósfera.

\section{A. Ciclo estacional}

El ciclo estacional del ozono es una variación senoidal de un año de duración, producida por diversos factores que afectan a la producción y destrucción natural de ozono. La destrucción de ozono se produce mayormente por fotólisis, es decir, mediante absorción de radiación ultravioleta $-310 \mathrm{~nm}$ o $1100 \mathrm{~nm}^{-}$, mientras que la producción se realiza por la fotólisis del oxígeno molecular a $240 \mathrm{~nm}$ y mediante la acción de choques moleculares. Para un dado nivel de radiación ultravioleta solar, estas dos acciones (producción y destrucción) llegan a un equilibrio. Durante los meses de verano, la cantidad de luz solar diaria, y por lo tanto, la radiación ultravioleta de onda larga que arriba a las capas inferiores de la atmósfera, es superior a la que se registra en los meses invernales. Otros factores que inciden en este ciclo son el ángulo de incidencia de la radiación solar, ya que producirán una atenuación mayor o menor; y la cantidad y tamaño de los aerosoles que también absorben la radiación solar. Por otra parte, cabe recordar que el ozono también varía con la latitud: crece desde el Ecuador hacia los polos. En el hemisferio sur, este crecimiento se da hasta los $55^{\circ}$, para luego decrecer un poco hacia el polo. Esta disminución se debe a procesos dinámicos característicos de esta zona, en contraposición con el hemisferio norte, debido particularmente por la distribución asimétrica del agua y de la tierra en el planeta [11].

\section{B. Ciclo solar}

El flujo radiado por el Sol varía de forma gradual día a día, y responde al número de manchas solares presente en el disco solar, cuyo ciclo es de 11 años. Al variar la intensidad de radiación solar ultravioleta que arriba al planeta se producirá, en consecuencia, una variación en la columna total de ozono. Esta influencia es mayor en las capas superiores de la atmósfera que en las inferiores. Por lo tanto, y como podrá apreciarse más adelante, durante el máximo del ciclo solar se produce un aumento en la tasa de producción de ozono respecto del proceso destructivo y viceversa [11].

\section{Oscilación cuasi-bienal}

Existe una variación en la columna total de ozono debido a una onda planetaria de casi 28 meses de período, denominada oscilación cuasi-bienal (QBO), que modula el sentido de los vientos en la estratósfera inferior [12]. Este ciclo se mide usando los vientos a 30 mbar desde Singapur. Se puede comprobar que las variaciones del ozono ecuatorial están en fase con los vientos medidos en Singapur; mientras que para $50^{\circ}$ de latitud, el desfasaje alcanza los $180^{\circ}$ aproximadamente [8]. Dicho lo anterior, el objetivo perseguido por el trabajo discutido aquí fue obtener datos de columna total de ozono para la ciudad de Puno y realizar un análisis del comportamiento.

\section{Materiales y métodos}

A falta de medidas experimentales de la columna total de ozono en nuestras latitudes, es preciso recurrir a otros métodos que proporcionen una estimación del valor diario de la columna total de ozono para el día en vigor. En este sentido, está ampliamente extendida la utilización del dato de la columna total de ozono medido con el sensor EPTOMS, disponible en la dirección https://ozoneaq.gsfc.nasa.gov/tools/ozonemap/. 
En dicho espacio es posible encontrar la cantidad total de ozono en columna sobre cualquier punto de la Tierra para la mayoría de las fechas comprendidas entre noviembre de 1978 y diciembre de 1994, y desde agosto de 1996 hasta la actualidad [9, 10]. Para acceder a esa información, basta con hacer clic en el mapa o ingresar la longitud y latitud geográfica del lugar donde se quiera calcular la columna total de ozono, medido en UD. Así entonces, en el desarrollo del trabajo que nos ocupa se obtuvieron los datos de la columna total de ozono para el periodo 2005-2017 reportados por el sensor Eptoms de la NASA en la ciudad de Puno $\left(15.83^{\circ}\right.$ latitud Sur y $70.03^{\circ}$ longitud Oeste), con una resolución espacial menor: $1.0^{\circ} \times 1.0^{\circ}[13]$.

\section{Resultados y discusiones}

Los datos obtenidos mediante el sensor Eptoms de la NASA respecto de la TOC se muestran en las figuras 1-13 de los años 2005 a 2017 en UD por meses. Se observa un comportamiento senoidal, en el que los valores máximos se observan en los meses de agosto, septiembre y octubre; mientras que se encuentran valores menores en los meses de febrero, marzo, abril -con lo que se obtiene un ecuación senoidal-.

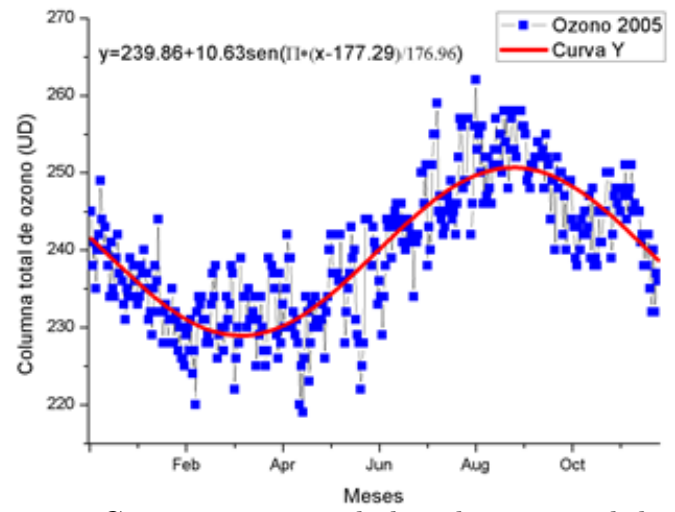

Figura 1. Comportamiento de la columna total de ozono para la ciudad de Puno durante el año 2005

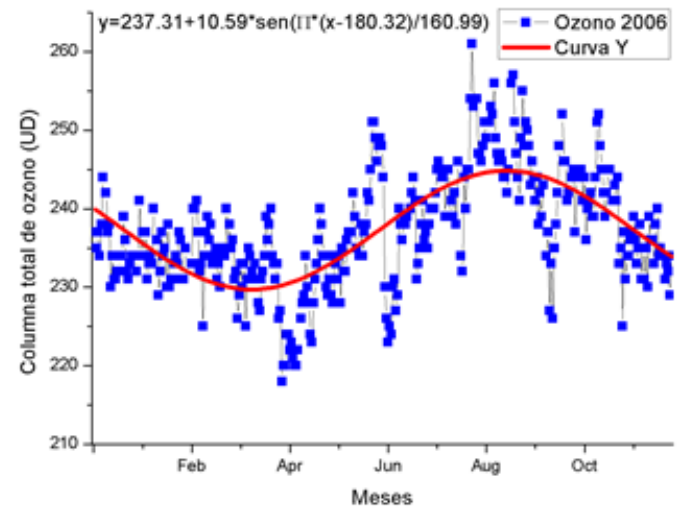

Figura 2. Comportamiento de la columna total de ozono para la ciudad de Puno durante el año 2006

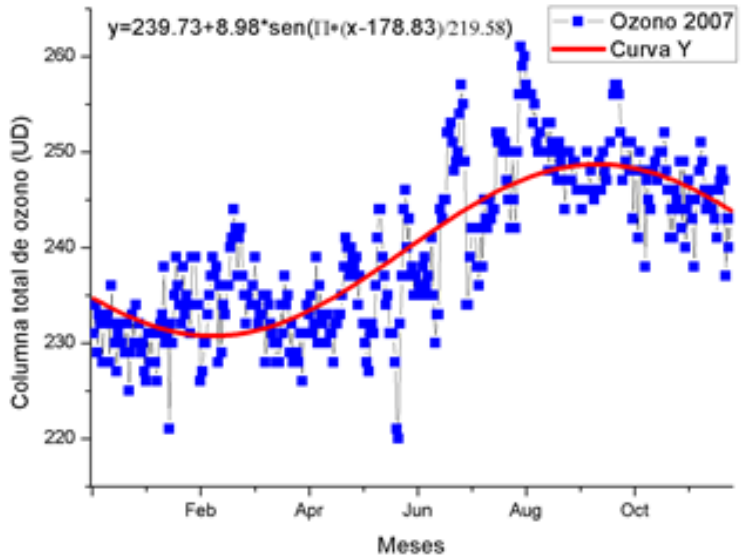

Figura 3. Comportamiento de la columna total de ozono para la ciudad de Puno durante el año 2007

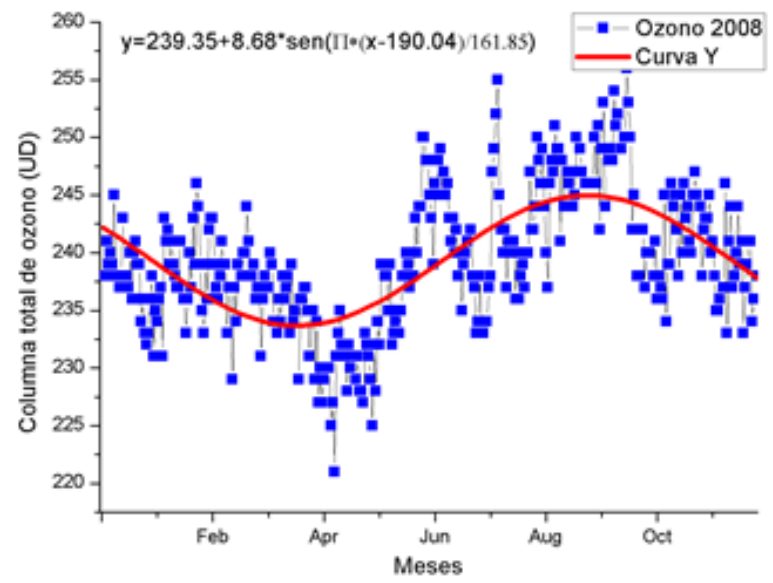

Figura 4. Comportamiento de la columna total de ozono para la ciudad de Puno durante el año 2008

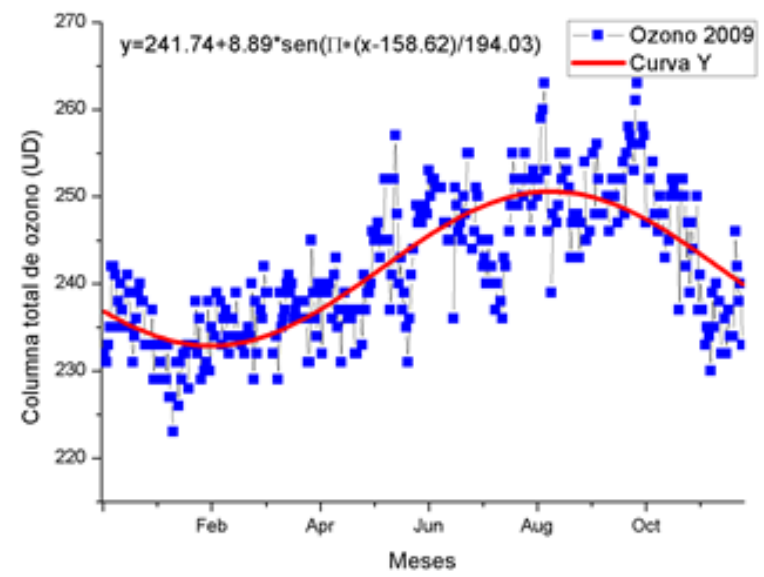

Figura 5. Comportamiento de la columna total de ozono para la ciudad de Puno durante el año 2009 


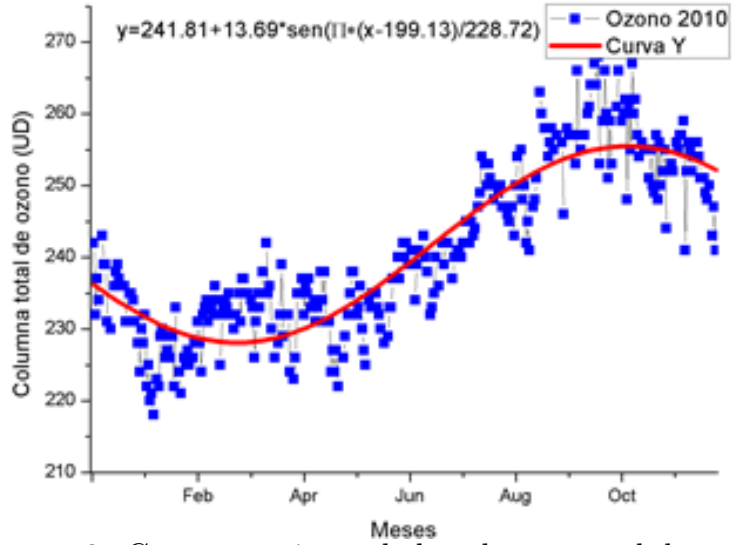

Figura 6. Comportamiento de la columna total de ozono para la ciudad de Puno durante el año 2010

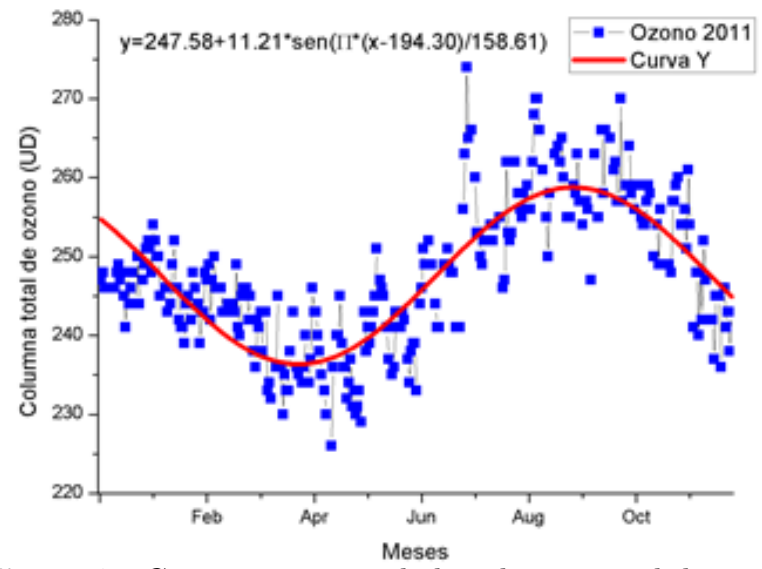

Figura 7. Comportamiento de la columna total de ozono para la ciudad de Puno durante el año 2011

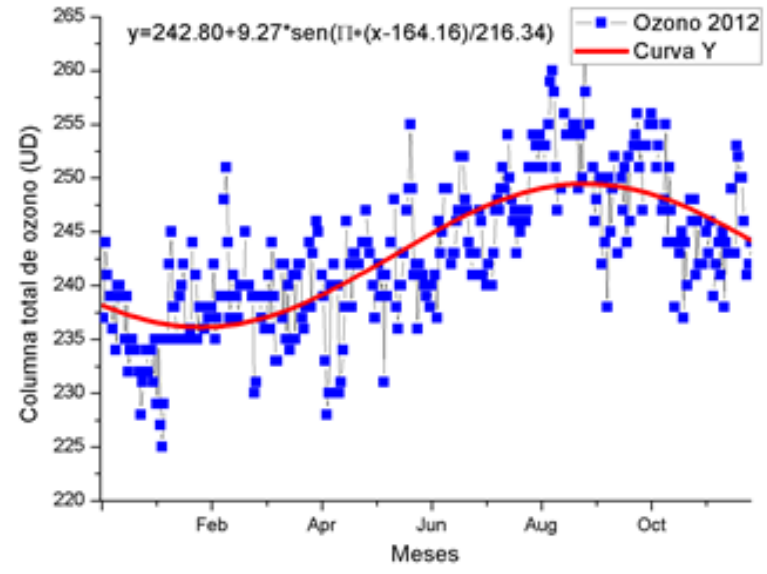

Figura 8. Comportamiento de la columna total de ozono para la ciudad de Puno durante el año 2012

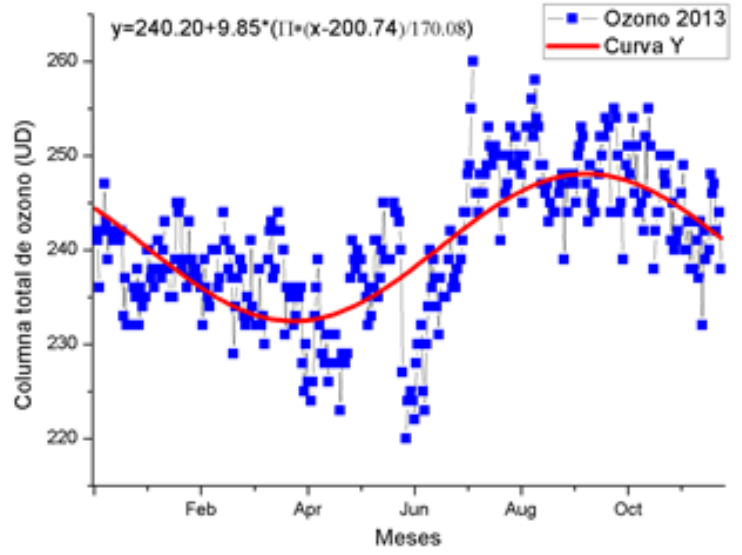

Figura 9. Comportamiento de la columna total de ozono para la ciudad de Puno durante el año 2013

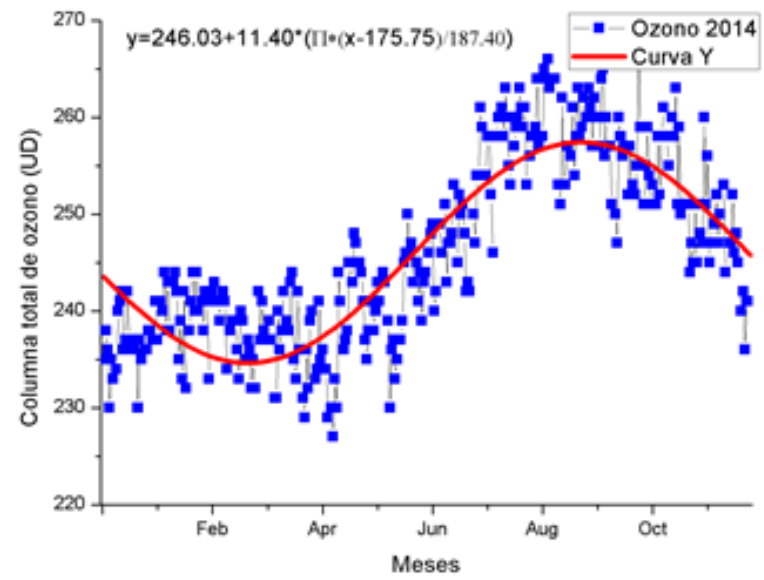

Figura 10. Comportamiento de la columna total de ozono para la ciudad de Puno durante el año 2014

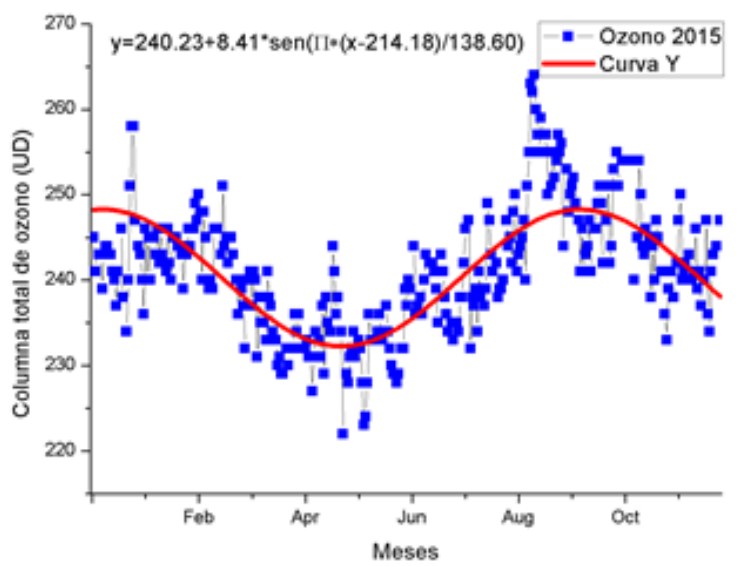

Figura 11. Comportamiento de la columna total de ozono para la ciudad de Puno durante el año 2015 


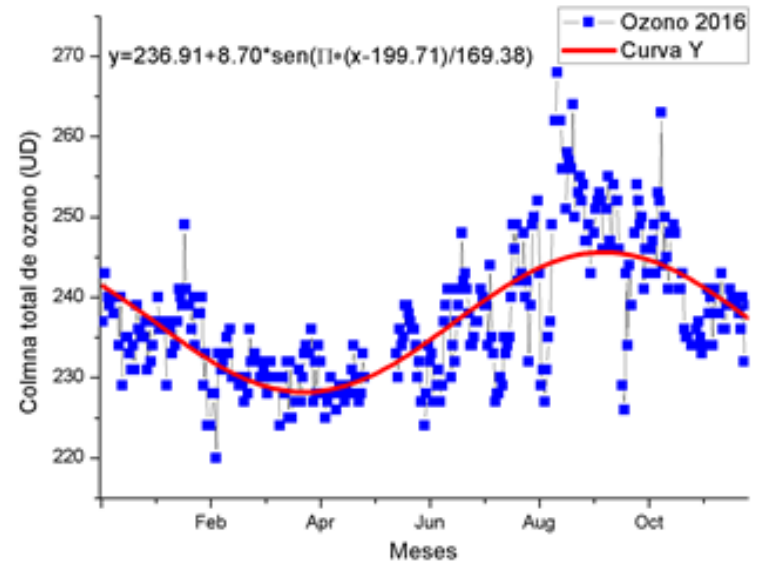

Figura 12. Comportamiento de la columna total de ozono para la ciudad de Puno durante el año 2016

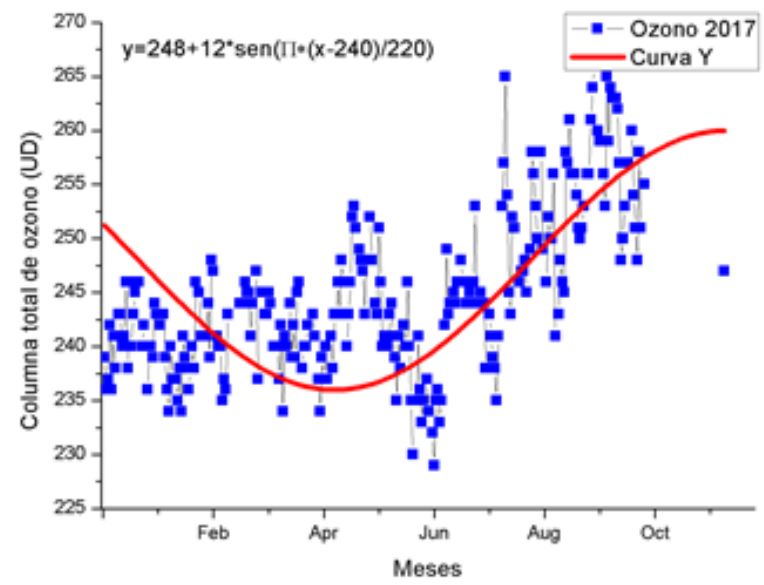

Figura 13. Comportamiento de la columna total de ozono para la ciudad de Puno durante el año 2017

La NASA utiliza un valor de referencia de 220 UD de columna total de ozono. Este fue elegido como el punto de partida para las observaciones de la Antártida en relación con el agujero de ozono, ya que no se encontraron valores de menos de 220 UD antes de 1979. Además, a partir de mediciones directas sobre la Antártida, un nivel de la columna de ozono de menos de 220 UD es un resultado de la pérdida de ozono a partir de cloro y bromo compuestos $[14,15]$.

Los valores máximos, mínimos y promedio de la TOC en UD se muestran en la tabla 1. Se registraron valores de 219 UD de TOC en mayo de 2005; de 218 UD en abril de 2006, y de 218 UD en febrero de 2010. Nótese que estos valores son inferiores a $220 \mathrm{UD}$, utilizado como referencia por NASA para considerarlos agujeros de ozono [16].
Tabla 1. Valores máximos, mínimos y promedio por años

\begin{tabular}{llll}
\hline \hline Año & Máximo & Mínimo & Promedio \\
\hline 2005 & 262 UD & 219 UD & 239.87 UD \\
2006 & 261 UD & 218 UD & 237.23 UD \\
2007 & 261 UD & 220 UD & 239.62 UD \\
2008 & 256 UD & 221 UD & 239.25 UD \\
2009 & 263 UD & 223 UD & 241.80 UD \\
2010 & 268 UD & 218 UD & 240.95 UD \\
2011 & 274 UD & 226 UD & 247.41 UD \\
2012 & 262 UD & 225 UD & 243.06 UD \\
2013 & 260 UD & 220 UD & 240.40 UD \\
2014 & 266 UD & 227 UD & 246.07 UD \\
2015 & 264 UD & 222 UD & 241.38 UD \\
2016 & 268 UD & 220 UD & 237.32 UD \\
2017 & 266 UD & 229 UD & 244.71 UD \\
\hline
\end{tabular}

En la figura 14 se muestra la columna total de ozono para el periodo 2005-2017 en función del número juliano. Obsérvese en la sobreposición de los datos el comportamiento de una función senoidal como las obtenidas en las figuras 1-13; en estos puede verse de los ajustes que el desfasaje varía año tras año.

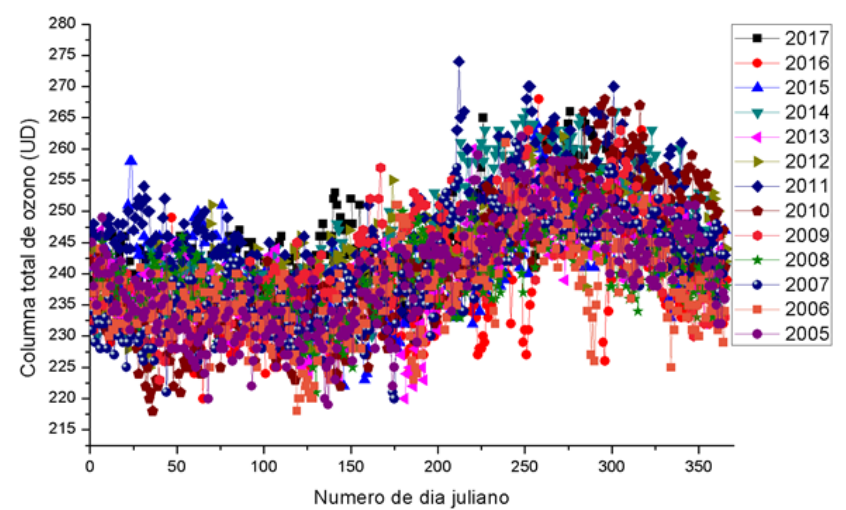

Figura 14. Comportamiento de la TOC para la ciudad de Puno, con respecto al número de día juliano

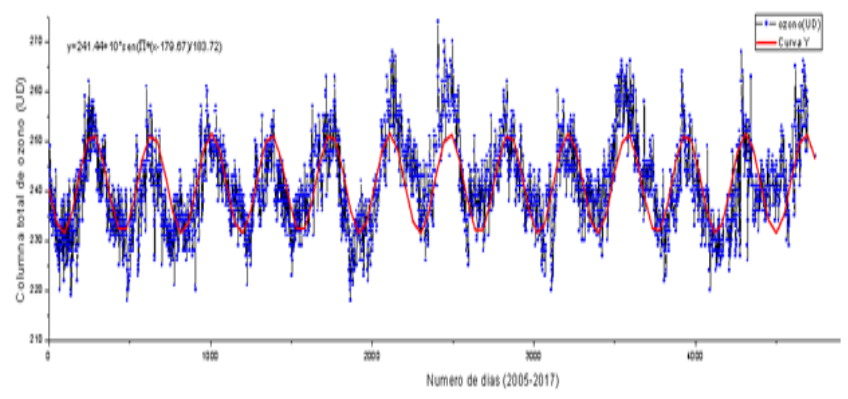

Figura 15. Comportamiento de la columna total de ozono para la ciudad de Puno para el periodo 2005-2017

Del análisis de datos de la TOC por años (Figura 15) se observa el comportamiento cíclico y senoidal de esta última durante el periodo estudiado. 


\section{Conclusiones}

Como se ha establecido hasta aquí, en el marco del presente trabajo se analizaron los datos reportados por el sensor Eptoms de la NASA para la latitud y longitud de la ciudad de Puno, con la finalidad de obtener valores de la TOC máxima, mínima y promedio. El análisis de la TOC por años muestra que el 17 de mayo de 2005, el 5 de febrero de 2010 y el 29 de abril de 2016 se registraron valores de TOC inferiores a los establecidos por la NASA: 219 UD en la primera fecha y 218 UD para la segunda y la tercera.

Por otra parte, el análisis de la columna total de ozono para el periodo comprendido entre 2005 y 2017 muestra un comportamiento senoidal, como lo establece el ciclo estacional. El estudio realizado, que comprendió un periodo de 13 años consecutivos, no permite observar la variación por el ciclo solar; para hacerlo sería necesario realizar un análisis de un periodo no inferior a 22 años consecutivos (puesto que, como se mencionó, su ciclo es de 11 años).

\section{Referencias}

[1] C. Sordo and C. Gutiérrez, "Cáncer de piel y radiación solar: experiencia peruana en la prevención y detección temprana del cáncer de piel y melanoma," Revista Peruana de Medicina Experimental y Salud Pública, vol. 30, pp. 113-117, 2013.

[2] A. Calle, A. Pérez, and J. Casanova, "Estudio y análisis de la irradiancia eritemática a partir de datos de ozono toms," Revista de Teledetección, vol. 9, 1998.

[3] K. M. Hernández Espinoza, J. Wright Gilmore, R. Morera, et al., "Medición y cálculo del índice ultravioleta en costa rica," pp. 41-55, 2014.

[4] M. Marín, I. Gómez, F. Tena, and M. Estrela, "Comparación de los datos de ozono obtenidos a partir de los satélites omi, sciamachy y gome2. idoneidad para la predicción del índice uv en valencia," in Proceedings of the XIII Congreso de la Asociación Española de Teledetección. Teledetección, Agua y desarrollo sostenible, Calatayud (Zaragoza), Spain, pp. 357-360, 2009.

[5] M. Huillca, "modelo de la radiación solar uv-b para la ciudad de puno," Revista Investigaciones Altoandinas, vol. 15, no. 1, pp. 55-63, 2013.

[6] M. Huillca Arbieto, C. William Taipe, and M. Saavedra, "Índice ultravioleta en la ciudad de puno para cielos claros," Revista de Investigaciones Altoandinas, vol. 19, no. 2, pp. 211-218, 2017.

[7] M. Rivas, E. Rojas, J. Cortés, and E. Santander, "Efecto de la altura en la radiación solar ultravioleta en arica norte de chile," Revista Facultad de Ingeniería-Universidad de Tarapacá, vol. 10, pp. 59-62, 2002.

[8] C. Puliafito, S. E. Puliafito, J. L. Quero, and M. A. Gantuz, "Análisis de la variación de la columna total de ozono en la región de mendoza en el período 1978-2000 y de los perfiles de ozono estratosféricos durante el período 1993-2000," Revista de la Universidad de Mendoza., 2002.

[9] A. M. Thompson, J. C. Witte, C. Sterling, A. Jordan, B. J. Johnson, S. J. Oltmans, M. Fujiwara, H. Vömel, M. Allaart, A. Piters, et al., "First reprocessing of southern hemisphere additional ozonesondes (shadoz) ozone profiles (1998-2016): 2. comparisons with satellites and ground-based instruments," Journal of Geophysical Research: Atmospheres, vol. 122, no. 23, pp. 13-000, 2017.

[10] R. S. Stolarski, P. Bloomfield, R. D. McPeters, and J. R. Herman, "Total ozone trends deduced from nimbus 7 toms data," Geophysical Research Letters, vol. 18, no. 6, pp. 1015-1018, 1991.

[11] P. Fabian, Atmosphäre und Umwelt Chemische Prozesse. Menschliche Eingriffe. Ozon-Schicht. Luftverschmutzung Smog. Saurer Regen. New York: Springer, 1992.

[12] M. Baldwin, L. Gray, T. Dunkerton, K. Hamilton, P. Haynes, W. Randel, J. Holton, M. Alexander, I. Hirota, T. Horinouchi, et al., "The quasi-biennial oscillation," Reviews of Geophysics, vol. 39, no. 2, pp. 179-229, 2001.

[13] J. P. Veefkind, J. F. de Haan, E. J. Brinksma, M. Kroon, and P. F. Levelt, "Total ozone from the ozone monitoring instrument (omi) using the doas technique," IEEE transactions on geoscience and remote sensing, vol. 44, no. 5, pp. 1239-1244, 2006.

[14] M. López Bartolomé, J. Montero Cadalso, J. M. San Atanasio, and A. Cansado, "Pérdida record de ozono estratosférico en el ártico durante la primavera de 2011: implicaciones sobre españa," 2011.

[15] "Nasa ozone watch: Latest status of ozone." [En línea]. Disponible en: https://ozonewatch.gsfc. nasa.gov/. [Accedido: 23-Jun-2019].

[16] "Nasa ozone watch: Ozone hole facts." [En línea]. Disponible en: https://ozonewatch.gsfc.nasa.gov/ facts/hole_SH.html. [Accedido: 23-Jun-2019]. 